\title{
Full-Duplex MIMO Small-Cells: Secrecy Capacity Analysis
}

\author{
Ayda Babaei*, A. Hamid Aghvami*, Arman Shojaeifard ${ }^{\dagger}$, and Kai-Kit Wong ${ }^{\dagger}$ \\ *Department of Informatics, King's College London, London WC2R 2LS, United Kingdom \\ ${ }^{\dagger}$ Electronic \& Electrical Engineering Department, University College London, London WC1E 7JE, United Kingdom \\ E-Mail: *ayda.babaei@kcl.ac.uk; *hamid.aghvami@kcl.ac.uk; ${ }^{\dagger}$ a.shojaeifard@ucl.ac.uk; ${ }^{\dagger}$ kai-kit.wong@ucl.ac.uk.
}

\begin{abstract}
This paper studies the physical (PHY)-layer security performance in full-duplex (FD) multiple-input multipleoutput (MIMO) small-cell networks. Here, we take into account (i) residual self-interference (SI) over Rician fading channels, and (ii) mutual-interference (MI) under successive interference cancellation (SIC) mechanism. Considering linear zero-forcing (ZF) beamforming, the downlink (DL) and uplink (UL) average secrecy rates under both scenarios of passive and colluding eavesdropping are derived. Our findings indicate that the FD functionality can provide substantial improvements in the PHYlayer security performance, especially with the aid of MIMO communications and interference cancellation solutions.
\end{abstract}

\section{INTRODUCTION}

Full-duplex (FD) communications is an emerging disruptive solution for improving the achievable spectral efficiency (SE) [1]. This trend is in line with the recent major breakthroughs in self-interference (SI) cancellation [2], [3]. Moreover, the mutual-interference (MI), a main limiting factor in largescale FD systems, can be tackled via successive interference cancellation (SIC) [4]. With the aid of state-of-the-art schemes for tackling SI and MI, significant FD versus half-duplex (HD) $\mathrm{SE}$ gains can be achieved in cellular networks [5], [6].

Physical (PHY)-layer security is a de facto requirement for the safeguarding of future wireless communications systems [7]. This topic has received a great deal of attention in recent years (see, e.g., [8] and the references therein for a survey). Given that the locations of the eavesdroppers (EDs) are in general not known to the cellular network operator, they can be modeled using point processes, see, e.g., [9]. More recently, the impact of randomly-located cooperating (i.e., colluding) EDs has also been investigated in [10].

In [11], a new solution for improving the secrecy capacity using FD transceivers which perform joint reception and jamming was proposed. The work in [11], as well as other related papers such as [12], were based on a deterministic single-cell setup. On the other hand, the PHY-layer security performance in large-scale FD systems is not well understood. In this work, we aim to bridge the gap by providing an analysis of average secrecy rate performance in FD multipleinput multiple-output (MIMO) small-cell networks.

Here, we employ the Poisson point process (PPP)-based abstraction model of multi-antenna base stations (BSs), user equipments (UEs), and EDs. The residual SI channels follow from the Rician distribution with arbitrary statistics. We consider SIC mechanism at the UE side, via imposing an arbitrary radius guard region in the respective $\mathrm{MI}$ expression. By leveraging on the tools from stochastic geometry, we characterize the downlink (DL) and uplink (UL) average secrecy rates and derive the statistics of the different useful and interference signals in closed-form. The validity of the theoretical findings is confirmed through Monte-Carlo (MC) simulations.

Notation: $\boldsymbol{X}$ is a matrix; $\boldsymbol{x}$ is a vector; $T, \dagger$, and + are the transpose, Hermitian, and pseudo-inverse; $\mathbb{E}_{x}[$.$] is the$ expectation; $\operatorname{Pr}[$.$] is the probability; \mathcal{F}_{x}[$.$] is the cumulative$ distribution function $(\mathrm{CDF}) ; \mathcal{P}_{x}[$.$] is the probability density$ function (PDF); $\mathcal{L}_{x}[$.$] is the Laplace transform (LT) function;$ $[x]^{+}=\max (x, 0)$ is the Ramp function; $\mathbf{I}_{(.)}$is the identity matrix; $\mathcal{C} \mathcal{N}\left(\mu, \nu^{2}\right)$ is the complex Gaussian distribution with mean $\mu$ and variance $\nu^{2} ; \Gamma($.$) and \Gamma(.,$.$) are the Gamma$ and incomplete Gamma functions; $\mathcal{G}(\kappa, \theta)$ is the Gamma distribution with shape parameter $\kappa$ and scale parameter $\theta$; and ${ }_{2} F_{1}(., . ; . ;$.$) is the Gauss hypergeometric function.$

\section{SySTEM DESCRIPTION}

Consider a large-scale multi-user MIMO small-cell network where the FD BSs and half-duplex (HD) UEs are deployed based on PPPs $\phi_{d}$ and $\phi_{u}$ with densities $\lambda_{d}$ and $\lambda_{u}$, respectively. Each FD small-cell BS, equipped with $N_{d}$ transmit and $N_{u}$ receive antennas, is considered to simultaneously serve $K_{d}$ DL and $K_{u}$ UL HD single-antenna UEs per resource block. Moreover, the EDs are modeled according to a homogeneous PPP $\phi_{e}$ with spatial density $\lambda_{e}$. Moreover, we consider the different scenarios where the (single-antenna) EDs are operating independently and cooperatively. Note that with obvious adjustments, the HD system, where the DL and UL occur over different resource blocks, can be described.

By invoking the Slivnyak's theorem, we perform the DL analysis for a typical HD UE $o$ located at the center. Let $l \in \phi_{d}, k \in \phi_{u}$, and $j \in \phi_{e}$ denote the locations of the $\mathrm{BS} l$, UL UE $k$, and $\mathrm{ED} j$, respectively. We consider the cellular association strategy based on the maximum received signal-to-interference-plus-noise ratio (SINR). Mathematically, the tagged BS of the reference DL UE $o$ satisfies $b=\arg \min \left(r_{l, o}\right), l \in \phi_{d}$, where $r_{l, o}=\|l-o\|$ denotes the Euclidean distance. The UL analysis, is carried out at the tagged BS $b$ with respect to the signal of an arbitrary HD UL UE $i$. Due to the cellular association procedure, the scheduled UEs locations are interdependent. Conditioning on the spatial constraints, we assume that the scheduled UEs 
follow from a homogeneous PPP [13]. Further, we consider the most malicious EDs in the DL and UL, respectively denoted with $v$ and $c$, which receive the strongest SINRs.

Let $p_{d}$ and $p_{u}$ denote the (per-user) BS and UE transmit powers, respectively. The DL channel gains from the BS $l$ at the UE $k$ and ED $j$ are $\boldsymbol{g}_{l, k} \in \mathcal{C}^{1 \cdot N_{d}}$ and $\boldsymbol{g}_{l, j} \in \mathcal{C}^{1 \cdot N_{d}}$, respectively. We denote the UL channel gains from the UE $k$ at the BS $l$ and ED $j$ using $\boldsymbol{h}_{k, l} \in \mathcal{C}^{N_{u} \cdot 1}$ and $h_{k, j}$, respectively. The cross-mode channel gains from the BS $l$ at the BS $b$, and from the UE $k$ at the UE $o$ are denoted with $\boldsymbol{H}_{l, b} \in \mathcal{C}^{N_{u} \cdot N_{d}}$ and $g_{k, o}$, respectively. The residual SI channel gain at the BS $b$ is denoted with $\boldsymbol{H}_{b, b} \in \mathcal{C}^{N_{u} \cdot N_{d}}$. The residual SI channels are Rician distributed with independent and indentically distributed (iid.) $\mathcal{C N}\left(\mu, \nu^{2}\right)$ coefficients [14]. All other channels are considered to be Rayleigh distributed with iid. elements drawn from $\mathcal{C N}(0,1)$. In addition, we utilize the unbounded path-loss model with exponent $\alpha(>2)$.

We employ linear zero-forcing (ZF) beamforming for suppressing intra-cell interference in both the DL and the UL. Let $\boldsymbol{G}_{l}=\left[\boldsymbol{g}_{l, k}^{T}\right]_{1<k<K_{d}}^{T} \in \mathcal{C}^{K_{d} \cdot N_{d}}$ denote the collective DL channels from the BS $l$ to its $K_{d}$ DL UEs. At the BS $l$, the linear ZF precoding matrix $V_{l}$ is selected equal to the normalized columns of $\boldsymbol{G}_{l}^{+}=\boldsymbol{G}_{l}^{\dagger}\left(\boldsymbol{G}_{l} \boldsymbol{G}_{l}^{\dagger}\right)^{-1} \in \mathcal{C}^{N_{d} \cdot K_{d}}$. Moreover, let $\boldsymbol{H}_{l}=\left[\boldsymbol{h}_{k, l}\right]_{1 \leq k \leq K_{u}} \in \mathcal{C}^{N_{u} \cdot K_{u}}$ represent the collective UL channels at the $\mathrm{BS} l$ from its $K_{u}$ scheduled UL UEs. At the BS $l$, the linear postcoding ZF matrix $\boldsymbol{W}_{l}$ is selected equal to the normalized rows of $\boldsymbol{H}_{l}^{+}=\left(\boldsymbol{H}_{l}^{\dagger} \boldsymbol{H}_{l}\right)^{-1} \boldsymbol{H}_{l}^{\dagger} \in \mathcal{C}^{K_{u} \cdot N_{u}}$.

The received SINR in the DL at the UE $o$ is given by

$$
\gamma_{o}^{\mathrm{FD}}=\frac{X_{o}}{I_{o}^{d, d}+I_{o}^{u, d}+\sigma_{o}^{2}}
$$

where $X_{o}=p_{d} G_{b, o} r_{b, o}^{-\alpha}, I_{o}^{d, d}=\sum_{l \in \phi_{d} \backslash\{b\}} p_{d} G_{l, o} r_{l, o}^{-\alpha}$, $I_{o}^{u, d}=\sum_{k \in \phi_{u}} p_{u} G_{k, o} r_{k, o}^{-\alpha}$, and $\sigma_{o}^{2}$ is the noise variance. Under linear ZF precoding, it can be shown that $G_{b, o} \sim$ $\mathcal{G}\left(N_{d}-K_{d}+1,1\right)$. With the assumption that the other BSs precoding matrices have independent columns, we have $G_{l, o} \sim \mathcal{G}\left(K_{d}, 1\right)$. Moreover, $G_{k, o} \sim \mathcal{G}(1,1)$.

On the other hand, the SINR in the UL at the BS $b$ with respect to the signal from an arbitrary UL UE $i$ is given by

$$
\gamma_{i}^{\mathrm{FD}}=\frac{X_{i}}{I_{i}^{u, u}+I_{i}^{d, u}+I_{i}^{i}+\sigma_{i}^{2}}
$$

where $X_{i}=p_{u} H_{i, b} r_{i, b}^{-\alpha}, I_{i}^{u, u}=\sum_{k \in \hat{\phi}_{u}} p_{u} H_{k, b} r_{k, b}^{-\alpha}, I_{i}^{d, u}=$ $\sum_{l \in \phi_{d} \backslash\{b\}} p_{d} H_{l, b} r_{l, b}^{-\alpha}, I_{i}^{i}=p_{b} H_{b, b}, \hat{\phi}_{u}$ denotes the set of outer-cell scheduled UL UEs, and $\sigma_{i}^{2}$ is the noise variance.
Considering linear ZF postcoding, $H_{i, b} \sim \mathcal{G}\left(N_{u}-K_{u}+1,1\right)$. In addition, $H_{k, b} \sim \mathcal{G}(1,1)$, and via recalling the assumption of independent (column-wise) outer-cell precoding matrices, $H_{l, b} \sim \mathcal{G}\left(K_{d}, 1\right)$. Furthermore, the residual SI channel power gain over the MIMO Rician fading channel can be approximated as $H_{b, b} \sim \mathcal{G}(\kappa, \theta)$ where (3) and (4) [14].

We can define the SINR in the DL at the most malicious ED $v$ as

$$
\gamma_{v}^{\mathrm{FD}}=\frac{X_{v}}{I_{v}^{d, d}+I_{v}^{u, d}+\sigma_{v}^{2}}
$$

where $X_{v}=p_{d} G_{b, v} r_{b, v}^{-\alpha}, I_{v}^{d, d}=\sum_{l \in \phi_{d} \backslash\{b\}} p_{d} G_{l, v} r_{l, v}^{-\alpha}$, $I_{v}^{u, d}=\sum_{k \in \phi_{u}} p_{u} G_{k, v} r_{k, v}^{-\alpha}$, and $\sigma_{v}^{2}$ is the noise variance. The channel power gains are given by $G_{b, v} \sim \mathcal{G}(1,1)$, $G_{l, v} \sim \mathcal{G}\left(K_{d}, 1\right)$, and $G_{k, v} \sim \mathcal{G}(1,1)$.

Further, the SINR in the UL at the most malicious ED $c$ is given by

$$
\gamma_{c}^{\mathrm{FD}}=\frac{X_{c}}{I_{c}^{u, u}+I_{c}^{d, u}+\sigma_{c}^{2}}
$$

where $X_{c}=p_{u} H_{o, c} r_{o, c}^{-\alpha}, I_{c}^{u, u}=\sum_{k \in \phi_{u}} p_{u} H_{k, c} r_{k, c}^{-\alpha}, I_{c}^{d, u}=$ $\sum_{l \in \phi_{d}} p_{d} H_{l, c} r_{l, c}^{-\alpha}$, and $\sigma_{c}^{2}$ is the noise variance. Here, the channel power gains are characterized using $H_{o, c} \sim \mathcal{G}(1,1)$, $H_{k, c} \sim \mathcal{G}(1,1)$, and $H_{l, c} \sim \mathcal{G}\left(K_{d}, 1\right)$.

\section{SECRECY RATE ANALYSIS}

In this section, we analyze the PHY-layer security performance in FD multi-user MIMO small-cell networks under both scenarios of passive and colluding eavesdropping. Note that the average rates (in $\mathrm{b} / \mathrm{s} / \mathrm{Hz}$ ) of the reference $\mathrm{DL}$ and $\mathrm{UL}$ UEs, $o$ and $i$, over two resource blocks, are denoted with $C_{o}^{\mathrm{FD}}$ and $C_{i}^{\mathrm{FD}}$ in $\mathrm{FD}$ mode, and $C_{o}^{\mathrm{HD}}$ and $C_{i}^{\mathrm{HD}}$ in $\mathrm{HD}$ mode, respectively. Similarly, the average rates (in $\mathrm{b} / \mathrm{s} / \mathrm{Hz}$ ) of the most malicious DL and UL EDs, $v$ and $c$, over two resource blocks, are denoted with $C_{v}^{\mathrm{FD}}$ and $C_{c}^{\mathrm{FD}}$ in FD mode, and $C_{v}^{\mathrm{HD}}$ and $C_{c}^{\mathrm{HD}}$ in HD mode, respectively.

Remark 1. By invoking the Jensen's inequality, $\mathbb{E}[\max (X, Y)\} \geq \max \{\mathbb{E}\{X\}, \mathbb{E}\{Y\}]$, the bounded DL and UL average secrecy rates (considering FD BSs) are respectively given by $S_{o}^{\mathrm{FD}}=\left[C_{o}^{\mathrm{FD}}-C_{v}^{\mathrm{FD}}\right]^{+}$and $S_{i}^{\mathrm{FD}}=\left[C_{i}^{\mathrm{FD}}-C_{c}^{\mathrm{FD}}\right]^{+}$. Similarly, in the case of HD operation, the bounded DL and UL average secrecy rates are respectively given by $S_{o}^{\mathrm{HD}}=\left[C_{o}^{\mathrm{HD}}-C_{v}^{\mathrm{HD}}\right]^{+}$and $S_{i}^{\mathrm{HD}}=\left[C_{i}^{\mathrm{HD}}-C_{c}^{\mathrm{HD}}\right]^{+}$.

We proceed by deriving explicit expressions for the DL and UL UEs average rates under linear ZF beamforming. Note that the proofs are omitted due to space limitations.

$$
\begin{aligned}
& \kappa \triangleq \frac{K_{d}\left(N_{u}+1\right)\left(N_{d}-K_{d}+2\right)\left(\mu^{2}+\nu^{2}\right)^{2}}{\left(2 N_{u} N_{d}+\frac{K_{d}\left(N_{d}-K_{d}+2\right)}{N_{u}+1}\left(N_{u} N_{d}-N_{u}-N_{d}-1\right)\right) \mu^{4}+\left(N_{u}+1\right)\left(N_{d}+1\right) \nu^{2}\left(2 \mu^{2}+\nu^{2}\right)} \\
& \theta \triangleq \frac{\left(2 N_{u} N_{d}+\frac{K_{d}\left(N_{d}-K_{d}+2\right)}{\left(N_{d}+1\right)}\left(N_{u} N_{d}-N_{u}-N_{d}-1\right)\right) \mu^{4}+\left(N_{u}+1\right)\left(N_{d}+1\right) \nu^{2}\left(2 \mu^{2}+\nu^{2}\right)}{\left(N_{u}+1\right)\left(N_{d}-K_{d}+2\right)\left(\mu^{2}+\nu^{2}\right)}
\end{aligned}
$$


Theorem 1. The DL average rates (in $\mathrm{b} / \mathrm{s} / \mathrm{Hz}$ ) of the useful UE $o$ in the FD and HD small-cell networks over two resource blocks are given by

$$
\begin{aligned}
& C_{o}^{\mathrm{FD}}=\frac{4 \pi \lambda_{d}}{\ln (2)} \int_{0}^{\infty} \int_{0}^{\infty} \frac{1}{1+\gamma}\left(\sum _ { n = 0 } ^ { N _ { d } - K _ { d } } \left\{\frac{(-s)^{n}}{n !} \frac{\mathrm{d}^{n}}{\mathrm{~d} s^{n}} e^{-s \sigma_{o}^{2}}\right.\right. \\
& \left.\left.\cdot \mathscr{L}_{I_{o}^{d, d}}[s] \mathscr{L}_{I_{o}^{u, d}}[s]\right\}_{s=\frac{\gamma r^{\alpha}}{p_{d}}}\right) \mathrm{d} \gamma r e^{-\pi \lambda_{d} r^{2}} \mathrm{~d} r \\
& C_{o}^{\mathrm{HD}}=\frac{2 \pi \lambda_{d}}{\ln (2)} \int_{0}^{\infty} \int_{0}^{\infty} \frac{1}{1+\gamma}\left(\sum _ { n = 0 } ^ { N _ { d } - K _ { d } } \left\{\frac{(-s)^{n}}{n !} \frac{\mathrm{d}^{n}}{\mathrm{~d} s^{n}} e^{-s \sigma_{o}^{2}}\right.\right. \\
& \left.\left.\cdot \mathscr{L}_{I_{o}^{d, d}}[s]\right\}_{s=\frac{\gamma r^{\alpha}}{p_{d}}}\right) \mathrm{d} \gamma r e^{-\pi \lambda_{d} r^{2}} \mathrm{~d} r \\
& \mathscr{L}_{I_{o}^{d, d}}[s]=e^{-\pi \lambda_{d} r^{2}\left({ }_{2} F_{1}\left(K_{d},-\frac{2}{\alpha} ; 1-\frac{2}{\alpha} ;-\frac{s p_{d}}{r \alpha}\right)-1\right)} \\
& \mathscr{L}_{I_{o}^{u, d}}[s]=e^{-\pi K_{u} \lambda_{d} \varepsilon^{2}\left({ }_{2} F_{1}\left(1,-\frac{2}{\alpha} ; 1-\frac{2}{\alpha} ;-\frac{s p_{u}}{\varepsilon^{\alpha}}\right)-1\right)} .
\end{aligned}
$$

Remark 2. The guard region radius $\varepsilon$ in the $\mathrm{MI}$ expression in (10) can be set according to the SIC mechanism at the UE side. In particular, $\varepsilon=0$ corresponds to the worst-case without SIC capability.

Theorem 2. The UL average rates (in $\mathrm{b} / \mathrm{s} / \mathrm{Hz}$ ) of the useful $\mathrm{UE} i$ in the FD and HD small-cell networks over two resource blocks are given by

$$
\begin{aligned}
& C_{i}^{\mathrm{FD}}=\frac{4 \pi \lambda_{d}}{\ln (2)} \int_{0}^{\infty} \int_{0}^{\infty} \frac{1}{1+\gamma}\left(\sum _ { n = 0 } ^ { N _ { u } - K _ { u } } \left\{\frac{(-s)^{n}}{n !} \frac{\mathrm{d}^{n}}{\mathrm{~d} s^{n}} e^{-s \sigma_{i}^{2}}\right.\right.
\end{aligned}
$$

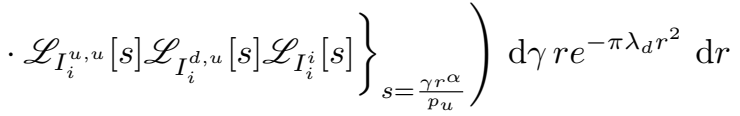

$$
\begin{aligned}
& C_{i}^{\mathrm{HD}}=\frac{2 \pi \lambda_{d}}{\ln (2)} \int_{0}^{\infty} \int_{0}^{\infty} \frac{1}{1+\gamma}\left(\sum _ { n = 0 } ^ { N _ { u } - K _ { u } } \left\{\frac{(-s)^{n}}{n !} \frac{\mathrm{d}^{n}}{\mathrm{~d} s^{n}} e^{-s \sigma_{i}^{2}}\right.\right.
\end{aligned}
$$

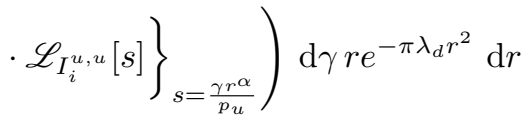

$$
\begin{aligned}
& \mathscr{L}_{I_{i}^{u}, u}[s]=e^{-\pi K_{u} \lambda_{d} r^{2}\left({ }_{2} F_{1}\left(1,-\frac{2}{\alpha} ; 1-\frac{2}{\alpha} ;-\frac{s p_{u}}{r^{\alpha}}\right)-1\right)} \\
& \mathscr{L}_{I_{i}^{d, u}}[s]=e^{-\pi \lambda_{d}\left(s p_{d}\right)^{\frac{2}{\alpha}} \frac{\Gamma\left(1-\frac{2}{\alpha}\right) \Gamma\left(K_{d}+\frac{2}{\alpha}\right)}{\Gamma\left(K_{d}\right)}} \\
& \mathscr{L}_{I_{i}^{i}}[s]=\left(1+s p_{d} \theta\right)^{-\kappa} .
\end{aligned}
$$

Remark 3. The Rician fading distribution parameters $\mu$ and $\nu$ in the residual SI expression in (15) can be tuned by design or measurements to capture the SI cancellation capability at the BS side. For example, $\nu=0$ and $\mu=0$ correspond to perfect SI removal and non line-of-sight (NLOS) SI, respectively.

Remark 4. The derivatives of the interfering terms LT functions required for the calculation of the average rates in the Theorems 1-2 can be readily computed through applying the Faà di Bruno's formula.

Next, we derive explicit expressions for the average rates of the most malicious passive EDs. Note that in this case the EDs act independently (do not exchange information).

Theorem 3. The DL average rates (in $\mathrm{b} / \mathrm{s} / \mathrm{Hz}$ ) of the most malicious passive ED $v$ in the FD and HD small-cell networks over two resource blocks are given by

$$
\begin{aligned}
& C_{v}^{\mathrm{FD}}=\frac{2}{\ln (2)} \int_{0}^{\infty} \frac{1}{1+\gamma}
\end{aligned}
$$

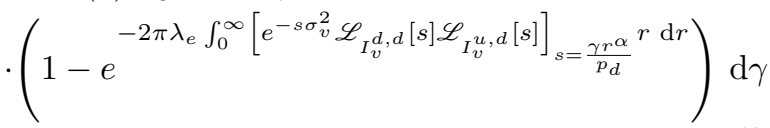

$$
\begin{aligned}
& C_{v}^{\mathrm{HD}}=\frac{1}{\ln (2)} \int_{0}^{\infty} \frac{1}{1+\gamma} \\
& \cdot\left(1-e^{-2 \pi \lambda_{e} \int_{0}^{\infty}\left[e^{-s \sigma_{v}^{2}} \mathscr{L}_{I_{v}^{d}, d}[s]\right]_{s=\frac{\gamma r^{\alpha}}{p_{d}}} r \mathrm{~d} r}\right) \mathrm{d} \gamma \\
& \mathscr{L}_{I_{v}^{d, d}}[s]=e^{-\pi \lambda_{d}\left(s p_{d}\right)^{\frac{2}{\alpha}} \frac{\Gamma\left(1-\frac{2}{\alpha}\right) \Gamma\left(K_{d}+\frac{2}{\alpha}\right)}{\Gamma\left(K_{d}\right)}}
\end{aligned}
$$

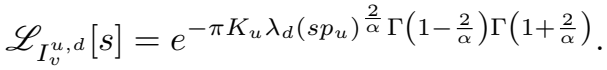

Theorem 4. The UL average rates (in $\mathrm{b} / \mathrm{s} / \mathrm{Hz}$ ) of the most malicious passive ED $c$ in the FD and HD small-cell networks over two resource blocks are given by

$$
\begin{aligned}
& C_{c}^{\mathrm{FD}}=\frac{2}{\ln (2)} \int_{0}^{\infty} \frac{1}{1+\gamma}
\end{aligned}
$$

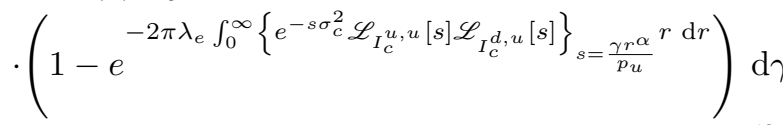

$$
\begin{aligned}
& C_{c}^{\mathrm{HD}}=\frac{1}{\ln (2)} \int_{0}^{\infty} \frac{1}{1+\gamma}
\end{aligned}
$$

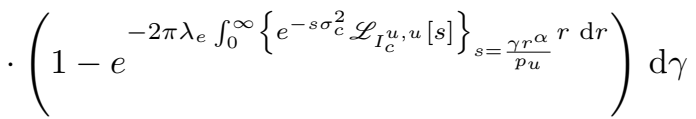

$$
\begin{aligned}
& \mathscr{L}_{I_{c}^{u, u}}[s]=e^{-\pi K_{u} \lambda_{d}\left(s p_{u}\right)^{\frac{2}{\alpha}} \Gamma\left(1-\frac{2}{\alpha}\right) \Gamma\left(1+\frac{2}{\alpha}\right)} \\
& \mathscr{L}_{I_{c}^{d, u}}[s]=e^{-\pi \lambda_{d}\left(s p_{d}\right)^{\frac{2}{\alpha}} \frac{\Gamma\left(1-\frac{2}{\alpha}\right) \Gamma\left(K_{d}+\frac{2}{\alpha}\right)}{\Gamma\left(K_{d}\right)}} .
\end{aligned}
$$

The average rates of the most malicious colluding EDs in the DL and UL are derived next. The cooperating EDs in this case form a distributed antenna system [10].

Theorem 5. The DL average rates (in $\mathrm{b} / \mathrm{s} / \mathrm{Hz}$ ) of the most malicious colluding $\mathrm{ED} v$ in the $\mathrm{FD}$ and $\mathrm{HD}$ small-cell 
networks over two resource blocks are given by

$$
\begin{aligned}
& C_{v}^{\mathrm{FD}} \leq \frac{4 \pi \lambda_{e} p_{d}}{\ln (2) \gamma(1+\gamma)} \int_{0}^{\infty} \int_{r_{0}}^{\infty} r^{1-\alpha} \int_{0}^{\infty} e^{-s \sigma_{v}^{2}} \\
& \text { - } \mathscr{L}_{I_{v}^{d, d}}[s] \mathscr{L}_{I_{v}^{u, d}}[s] \mathrm{d} s \mathrm{~d} r \mathrm{~d} \gamma \\
& C_{v}^{\mathrm{HD}} \leq \frac{2 \pi \lambda_{e} p_{d}}{\ln (2) \gamma(1+\gamma)} \int_{0}^{\infty} \int_{r_{0}}^{\infty} r^{1-\alpha} \int_{0}^{\infty} e^{-s \sigma_{v}^{2}} \\
& \text { - } \mathscr{L}_{I_{v}^{d, d}}[s] \mathrm{d} s \mathrm{~d} r \mathrm{~d} \gamma .
\end{aligned}
$$

Theorem 6. The UL average rates (in $\mathrm{b} / \mathrm{s} / \mathrm{Hz}$ ) of the most malicious colluding ED $c$ in the FD and HD small-cell networks over two resource blocks are given by

$$
\begin{aligned}
& C_{c}^{\mathrm{FD}} \leq \frac{4 \pi \lambda_{e} p_{u}}{\ln (2) \gamma(1+\gamma)} \int_{0}^{\infty} \int_{r_{0}}^{\infty} r^{1-\alpha} \int_{0}^{\infty} e^{-s \sigma_{c}^{2}} \\
& \text { - } \mathscr{L}_{I_{c}^{u, u}}[s] \mathscr{L}_{I_{c}^{d, u}}[s] \mathrm{d} s \mathrm{~d} r \mathrm{~d} \gamma \\
& C_{c}^{\mathrm{HD}} \leq \frac{2 \pi \lambda_{e} p_{u}}{\ln (2) \gamma(1+\gamma)} \int_{0}^{\infty} \int_{r_{0}}^{\infty} r^{1-\alpha} \int_{0}^{\infty} e^{-s \sigma_{c}^{2}} \\
& \text { - } \mathscr{L}_{I_{c}^{u, u}}[s] \mathrm{d} s \mathrm{~d} r \mathrm{~d} \gamma \text {. }
\end{aligned}
$$

Remark 5. The parameter $r_{0}$ in the colluding EDs average rates from Theorems 5-6 represents the guard region distance required to satisfy the secrecy non-outage condition.

\section{Numerical Results}

We assess the PHY-layer security performance of FD and HD MIMO small-cell networks in the presence of a Poisson field of EDs. The small-cell BS deployment density is $\lambda^{(d)}=\frac{4}{\pi}$ per $\mathrm{km}^{2}$. The (per-user) BS and UE transmit powers are $p_{d}=23 \mathrm{dBm}$ and $p_{u}=20 \mathrm{dBm}$, respectively. The noise spectral density at all receivers is $-170 \mathrm{dBm} / \mathrm{Hz}$ and the system bandwidth is $W=10 \mathrm{MHz}$. The path-loss exponent is $\alpha=4$. In the FD small-cell network, the DL and UL run simultaneously (with SIC and SI cancellation), whereas in the HD small-cell network, the DL and UL occur over different resource blocks. Note that all results correspond to the per-user average secrecy rate performance over two resource blocks.

\section{A. Impact of the Number of Base Station Antennas}

1) Passive Eavesdroppers: The impact of the number of BS antennas on the small-cell network PHY-layer security performance under passive eavesdropping is depicted in Fig. 1. It can be seen that the average secrecy rate increases in the number of antennas. Furthermore, the FD over HD smallcell network PHY-layer security performance gain increases in the number of antennas. In point of fact, even with SIC mechanism and perfect SI suppression, only negligible FD versus HD improvements in average secrecy performance can be achieved when the small-cell BSs are equipped with a few antennas. This trend highlights the essential role of MIMO in harnessing the full potential of FD technology.

2) Colluding Eavesdroppers: The impact of the number of BS antennas on the small-cell network PHY-layer security performance under colluding eavesdropping is depicted in Fig. 2. Similar to the case of passive EDs, increasing the
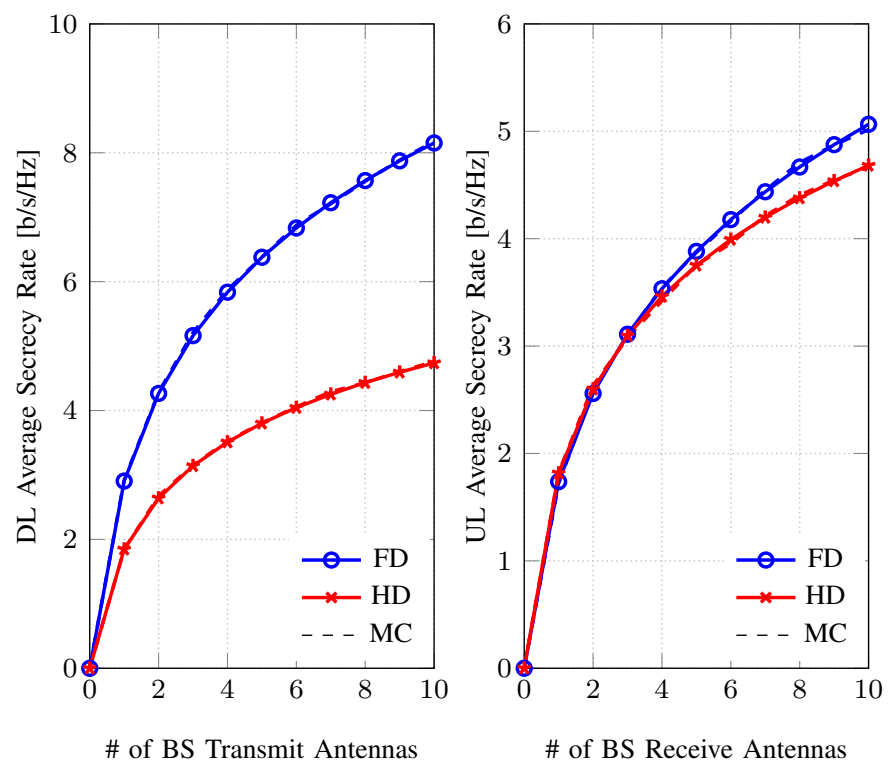

Fig. 1. Other parameters: $\lambda_{e}=\frac{\lambda_{d}}{10} \mathrm{~km}^{-2}, K_{d}=K_{u}=1$.
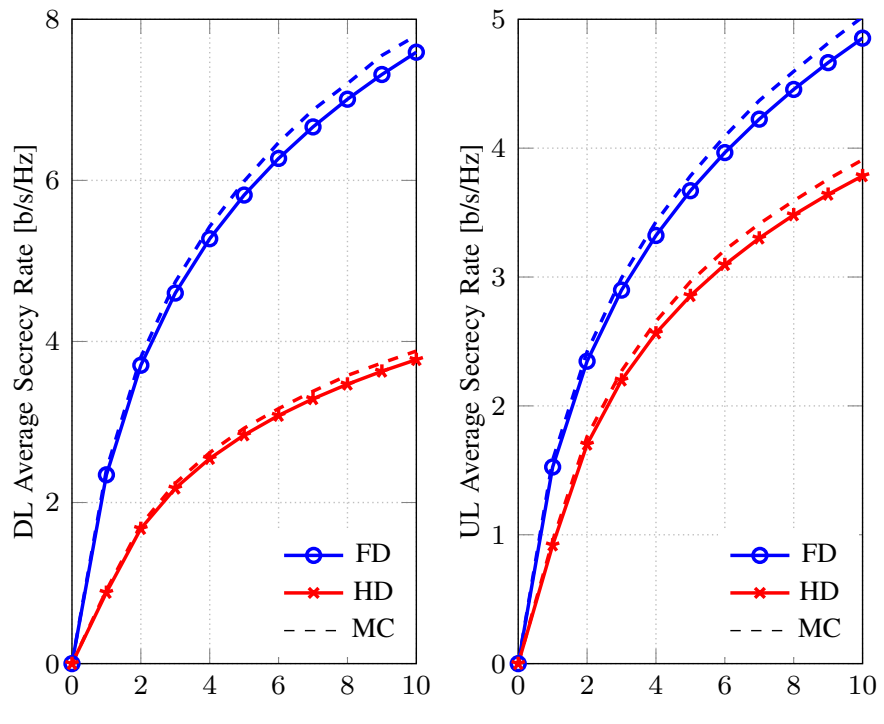

\# of BS Transmit Antennas

\# of BS Receive Antennas

Fig. 2. Other parameters: $\lambda_{e}=\frac{\lambda_{d}}{200} \mathrm{~km}^{-2}, K_{d}=K_{u}=1, r_{0}=\frac{1}{2} \mathrm{~km}$.

number of antennas always results in higher average secrecy rates, as well as greater FD versus HD PHY-layer security performance gains. Furthermore, our findings indicate that the relative FD versus HD average secrecy rate gain can be considerably higher in the case of colluding EDs. The reason is because each cooperative ED experiences added interference (i.e., MI) in the case of FD operation which in turn degrades the colluding EDs' combined SINR.

\section{B. Impact of the Eavesdropper Density}

1) Passive Eavesdroppers: The impact of the ED spatial density on the small-cell network PHY-layer security performance under passive eavesdropping is depicted in Fig. 3. As 

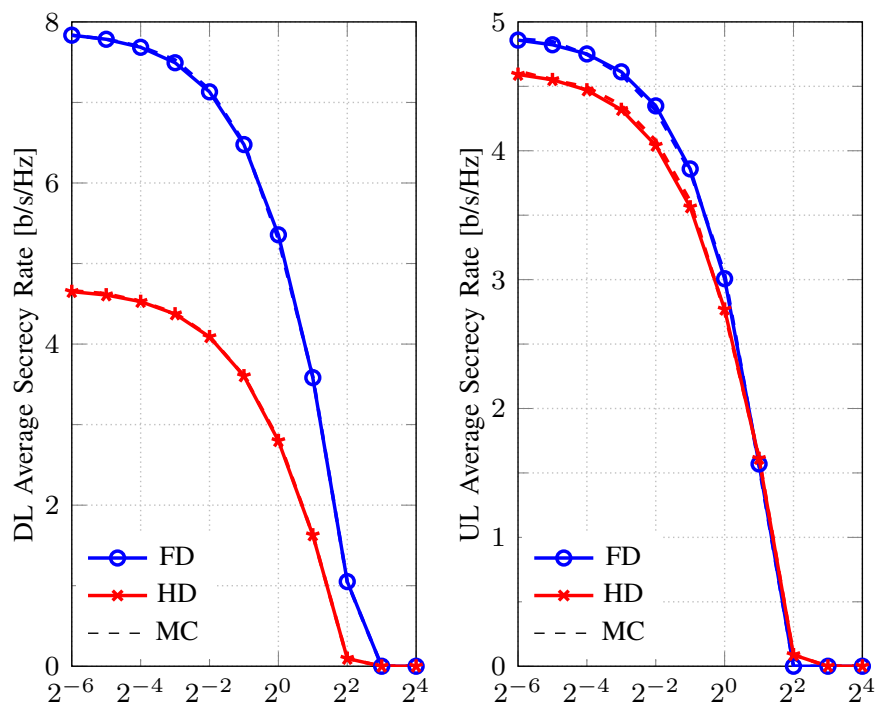

ED/BS Density Ratio [log base two] ED/BS Density Ratio [log base two]

Fig. 3. Other parameters: $N_{d}=N_{u}=8, K_{d}=K_{u}=1$.
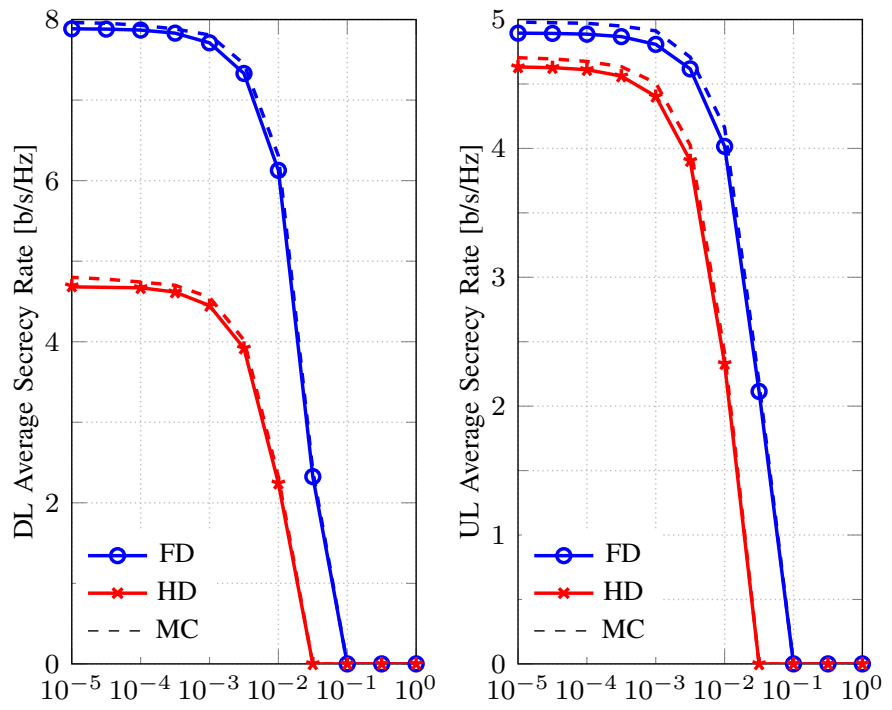

ED/BS Density Ratio [log base ten]

ED/BS Density Ratio [log base ten]

Fig. 4. Other parameters: $N_{d}=N_{u}=8, K_{d}=K_{u}=1, r_{0}=\frac{1}{2} \mathrm{~km}$.

expected, it can be seen that the average secrecy rate decreases in the ratio of ED over BS deployment densities. Moreover, increasing the spatial density of the EDs decreases the FD versus HD small-cell network PHY-layer security performance gain. This is because the stronger ED channel capacity degrades the average secrecy rate values, which in turn, reduces the gap between the PHY-layer security performance in the FD and HD small-cell networks.

2) Colluding Eavesdroppers: The impact of the ED spatial density on the small-cell network PHY-layer security performance under colluding eavesdropping is depicted in Fig. 4. We can observe similar trends to those highlighted in the case of passive EDs. Intuitively, the PHY-layer security performance benefits from having a smaller ED PPP-based deployment density. Furthermore, the underlying FD versus HD gains in terms of average secrecy rate increases with smaller values of $\lambda_{e}$. Note that the case of collusion represents the absolute worst-case scenario in terms of secrecy capacity. Therefore, unless the ED spatial density is very small, the small-cell network experiences secrecy outage with a high probability.

\section{CONCLUSIONS}

We analyzed the PHY-layer security performance in largescale FD multi-user MIMO small-cell networks. By leveraging on the tools from applied probability theory, we derived explicit expressions for the average secrecy rates under different passive and colluding eavesdropping scenarios. The findings highlighted that significant improvements in PHY-layer security performance can be achieved through FD enabled BSs, particularly, with the aid of MIMO communications and interference cancellation solutions.

\section{REFERENCES}

[1] J. I. Choi, M. Jain, K. Srinivasan, P. Levis, and S. Katti, "Achieving single channel, full duplex wireless communication," in Proc. MobiCom. ACM, 2010

[2] T. Riihonen, S. Werner, and R. Wichman, "Mitigation of loopback selfinterference in full-duplex MIMO relays," IEEE Trans. Signal Process., vol. 59, no. 12, pp. 5983-5993, Dec. 2011.

[3] E. Everett, A. Sahai, and A. Sabharwal, "Passive self-interference suppression for full-duplex infrastructure nodes," IEEE Trans. Wireless Commun., vol. 13, no. 2, pp. 680-694, Feb. 2014.

[4] C. Wang, H. Farhadi, and M. Skoglund, "Achieving the degrees of freedom of wireless multi-user relay networks," IEEE Trans. Commun., vol. 60, no. 9, pp. 2612-2622, Sept. 2012.

[5] D. Nguyen, L. N. Tran, P. Pirinen, and M. Latva-aho, "On the spectral efficiency of full-duplex small cell wireless systems," IEEE Trans. Wireless Commun., vol. 13, no. 9, pp. 4896-4910, Sept. 2014.

[6] A. Shojaeifard, K. K. Wong, M. D. Renzo, G. Zheng, K. A. Hamdi, and J. Tang, "Massive MIMO-enabled full-duplex cellular networks," IEEE Trans. Commun., accepted 2017.

[7] N. Yang, L. Wang, G. Geraci, M. Elkashlan, J. Yuan, and M. D. Renzo, "Safeguarding 5G wireless communication networks using physical layer security," IEEE Commun. Mag., vol. 53, no. 4, pp. 20-27, Apr. 2015.

[8] A. Mukherjee, S. A. A. Fakoorian, J. Huang, and A. L. Swindlehurst, "Principles of physical layer security in multiuser wireless networks: A survey," IEEE Commun. Surveys Tuts., vol. 16, no. 3, pp. 1550-1573, Third Quart. 2014.

[9] T. X. Zheng, H. M. Wang, J. Yuan, D. Towsley, and M. H. Lee, "Multiantenna transmission with artificial noise against randomly distributed eavesdroppers," IEEE Trans. Commun., vol. 63, no. 11, pp. 4347-4362, Nov. 2015.

[10] G. Chen, J. P. Coon, and M. D. Renzo, "Secrecy outage analysis for downlink transmissions in the presence of randomly located eavesdroppers," IEEE Trans. Inf. Forensics Security, vol. 12, no. 5, pp. 1195-1206, May 2017.

[11] G. Zheng, I. Krikidis, J. Li, A. P. Petropulu, and B. Ottersten, "Improving physical layer secrecy using full-duplex jamming receivers," IEEE Trans. Signal Process., vol. 61, no. 20, pp. 4962-4974, Oct. 2013.

[12] A. E. Shafie, A. Sultan, and N. Al-Dhahir, "Physical-layer security of a buffer-aided full-duplex relaying system," IEEE Commun. Lett., vol. 20, no. 9, pp. 1856-1859, Sept. 2016.

[13] M. D. Renzo and P. Guan, "Stochastic geometry modeling and systemlevel analysis of uplink heterogeneous cellular networks with multiantenna base stations," IEEE Trans. Commun., vol. 64, no. 6, pp. 24532476, June 2016.

[14] A. Shojaeifard, K. K. Wong, M. D. Renzo, G. Zheng, K. A. Hamdi, and J. Tang, "Self-interference in full-duplex multi-user MIMO channels," IEEE Commun. Lett., vol. 21, no. 4, pp. 841-844, Apr. 2017. 